\title{
Myoglobin-biomimetic electroactive materials made by surface molecular imprinting on silica beads and their use as ionophores in polymeric membranes for potentiometrictransduction
}

\author{
Felismina T.C. Moreira, Rosa A.F. Dutra, Joao P.C. Noronha, M. Goreti F. Sales
}

\begin{abstract}
Myoglobin $(\mathrm{Mb})$ is among the cardiac biomarkers playing a major role in urgent diagnosis of cardio- vascular diseases. Its monitoring in point-ofcare is therefore fundamental. Pursuing this goal, a novel biomimetic ionophore for the potentiometric transduction of Mb is presented. It was synthesized by surface molecular imprinting (SMI) with the purpose of developing highly efficient sensor layers for near-stereochemical recognition of $\mathrm{Mb}$. The template $(\mathrm{Mb})$ was imprinted on a silane surface that wascovalently attached to silica beads by means of self-assembled monolayers. First the silica was modified with an external layer of aldehyde groups. Then, $\mathrm{Mb}$ was attached by reaction with its amine groups (on the external surface) and subsequent formation of imine bonds. The vacant places surrounding $\mathrm{Mb}$ were filled by polymerization of the silane monomers $3-$ aminopropyltrimethoxysilane (APTMS) and propy- ltrimethoxysilane (PTMS). Finally, the template was removed by imine cleavage after treatment with oxalic acid. The results materials were finely dispersed in plasticized PVC selective membranes and used as ionophores in potentiometric transduction. The best analytical features were found in HEPES buffer of $\mathrm{pH} 4$. Under this condition, the limits of detection were of $1.3 \times 10^{-6} \mathrm{~mol} / \mathrm{L}$ for a linear response after

$8.0 \times 10^{-7} \mathrm{~mol} / \mathrm{L}$ with an anionic slope of $-65.9 \mathrm{mV} /$ decade. The imprinting effect was tested by prepar- ing non-imprinted (NI) particles and employing these materials as ionophores. The resulting membranes showed no ability to detect Mb. Good selectivity was observed towards creatinine, sacarose, fructose, galactose, sodium glutamate, and alanine. The analytical application was conducted successfully and showed accurate and precise results.
\end{abstract}

Keywords:

Surface molecular imprint, Sensor, Myoglobin, Cardiac biomarker, Potentiometry, Ion-selective electrodes

\section{Introduction}

Cardiovascular diseases are the leading cause of death around the world and include coronary heart disease, cerebrovascular disease, raised blood pressure, peripheral artery disease, rheumatic heart disease and congenital heart disease and heart failure (WHO, 2011). Symptoms of different acute coronary syndrome (ACS) conditions are very similar and include chest pain, pressure, shortness of breath, and/or nausea. These symptoms are typically associated with heart attacks and angina, but they may also be seen with non-heart-related conditions.
Since the treatment for each previous condition differs, and most of them require urgent measures, it is imperative that physicians are provided with additional information in a short time basis, enabling them to carry out quick and accurate diagnosis.

Cardiac biomarkers have been employed so far to achieve this goal. Cardiac biomarkers are substances that are released into the blood when there is damage to the heart muscle (Adams and Miracle, 1998). They are monitored by taking a blood sample when a patient has symptoms of ACS. There are several critical cardiac markers, whose levels vary along time (Plebani and Zaninotto, 1999). Although not cardiac-specific, Myoglobin (Mb) is one of the very early known markers that increase after acute myocardial infarction (Montague and Kircher, 1995), and its rapid screening under acute physiological conditions is fundamental. Human Mb has 153 amino acid residues in a highly folded and compact structure with eight separate and distinct alpha helical 

secondary structures and molecular weight $16.7 \mathrm{kDa}$. The dimension of Myoglobin is $(3 \mathrm{~nm} \times 4 \mathrm{~nm} \times 5 \mathrm{~nm})$ and the molar volume is $1.87 \times 104 \mathrm{~cm}^{3} \mathrm{~mol}^{-1}$ (Singh-Zocchi et al., 2002; Gunda and Mitra, 2010).

Several conventional methods have been employed to detect and quantify $\mathrm{Mb}$. These include enzyme linked immunosorbent assay (ELISA) (Sarkara and Mandala, 1985; Alan et al., 1992), and chromatographic (Powell et al., 1984; Mayer et al., 2006; Fresnea, 1964; Han et al., 1994; Maltseva et al., 1987; Kelner and Alexander, 1985) or spectrophotometric methods (Schuder et al., 1979; Modi et al., 1989; Boesken et al., 1977; Shiomi et al., 2005). In general, these methods lack the required specificity and/or involve several steps, are time consuming and require very expensive reagents.

Alternative methods are derived from immunochemical assays, taking advantage of the specific responses between the analyte $(\mathrm{Mb})$ and their natural receptors (antibodies). Several techniques have been developed in this context, including self-assembled monolayers (SAMs) using impedance and or capacitance measurements (Wanga et al., 2008; Billah et al., 2008; Zhang and Li, 2001; Li et al., 2009) and surface plasmon resonance (SPR) (Malone and Masson, 2005; Masson et al., 2007). However, these procedures keep long, expensive for routine analytical measurements and unable to be applied in point-of-care.

Replacing natural receptors by their artificial counterpartscould turn out an advantageous principle for $\mathrm{Mb}$ determination. The resulting materials hold many advantages over natural receptors. They offer higher stability in extreme conditions of $\mathrm{pH}$ and temperature, higher mechanical strength, lower cost, and reusability. Suitable Molecular Imprinting (MI) techniques may be selected to designa successfulartificial antibody for Mb. MI is a fast developing technology and has also been investigated for sensing applications, including those of potentiometric transduction (Moreira et al., 2010).

Potentiometric sensors offer the advantage of selectivity and portability for use in point-of-care and have been widely recognized as potential analytical tools in this field. The inherent method is simple, precise, accurate and inexpensive regarding reagent consumption and equipment involved. Washing steps arenot required before the signal can be read and the analyte can be captured from complex mixtures without prior purification. In terms of environmental "impact", they are called a green analytical approach for avoiding the use of organic solvents and discharging wastewaters of small concern with regard to volume and composition.

Thus, a potentiometric sensor selective to Myoglobin $(\mathrm{Mb})$ is proposed. Surface imprint of $\mathrm{Mb}$ on silica beads was selected for this purpose for presenting more efficient rebinding properties. Thecorresponding MI particles wereincluded in a polymeric membrane to behave as $\mathrm{Mb}$ ionophore. The imprinting effect was tested by carrying out the same procedure without template $(\mathrm{Mb})$. The non-imprinted (NI) particles so obtained were similarly included in polymeric membranes and used as control.

\section{Experimental}

\subsection{Apparatus}

Emf were measured in a Crison $\mu \mathrm{pH} 2002$ decimilivoltammeter $( \pm 0.1 \mathrm{mV}$ sensitivity), at room temperature, and under constant stirring by means of a Crison, microST 2038. The output signal was reconnected to a commutation point with six ways out, enabling the simultaneous reading of six selective electrodes.

The potentiometric cell was assembled as conductive contact of epoxy-graphite $\mid \mathrm{Mb}$ selective membrane|buffered sample solution (HEPES, $\left.1 \times 10^{-3} \mathrm{~mol} / \mathrm{L}, \mathrm{pH} 4.5\right) \mid$ electrolyte solution,
$\mathrm{KCl}|\mathrm{AgCl}(\mathrm{s})| \mathrm{Ag}$. Thereference was an Ag/ AgCldouble-junction electrode (Orion 90-02-00).

When necessary, the $\mathrm{pH}$ was measured by a Crison CWL/S7 combined glass electrode connected to a Crison decimilivoltammeter, pHmeter, GLP22.

\subsection{Reagents}

All chemicals were of analytical grade and de-ionized water (conductivity $<0.1 \mu \mathrm{S} / \mathrm{cm}$ ) was employed. Aminopropyl silica (particle size $15-40 \mu \mathrm{m}$, mean pore size $75 \AA$ ), $\mathrm{Mb}$, 4-(2-hydroxyethyl)-1-piperazineethanesulfonic acid (HEPES), piperazine-N, $\mathrm{N}^{\star}$-bis(2-ethanesulfonic acid) (PIPES), $o$-nitrophenyloctyl (oNPOE), poly(vinyl chloride) (PVC) of high molecular weight, APTMS, PTMS, glutaraldehyde, oxalic acid, and sodium chloride $(\mathrm{NaCl})$ were purchased from Fluka. Tetrahydrofuran (THF) was obtained from Riedel-deHäen.

\subsection{Solutions}

Stock solutions of $1.0 \times 10^{-4} \mathrm{~mol} / \mathrm{L} \mathrm{Mb}$ were prepared in water. Less concentrated $\mathrm{Mb}$ standards were prepared by accurate dilution of the previous solution in buffer. Buffer solutions were $1.0 \times 10^{-2} \mathrm{~mol} / \mathrm{L}$ HEPES. The $\mathrm{pH}$ of this solution was altered by adding suitable volumes of either concentrated hydrochloric acid or saturated sodium hydroxide solution freshly prepared.

The interference of other chemicals was evaluated for $1.0 \times 10^{-6} \mathrm{~mol} / \mathrm{L} \mathrm{Mb}$ prepared in $\mathrm{pH} 4.5$ buffer. Solutions of interfering species were of variable concentrations and prepared in the same buffer. Creatinine (Creat, $1.04 \times 10^{-3} \mathrm{~mol} / \mathrm{L}$ ), sucrose (Sac, $\left.1.61 \times 10^{-5} \mathrm{~mol} / \mathrm{L}\right)$, fructose (Fru, $\left.9.34 \times 10^{-4} \mathrm{~mol} / \mathrm{L}\right)$, galactose (Gat, $\left.5.58 \times 10^{-5} \mathrm{~mol} / \mathrm{L}\right)$, sodium glutamate $\left(\mathrm{Glt}, 7.61 \times 10^{-5} \mathrm{~mol} / \mathrm{L}\right)$, thiamine (Tia, $2.13 \times 10^{-5} \mathrm{~mol} / \mathrm{L}$ ), alanine (Ala, $6.17 \times 10^{-5} \mathrm{~mol} / \mathrm{L}$ ), and glucose (Gluc, $\left.4.19 \times 10^{-5} \mathrm{~mol} / \mathrm{L}\right)$ solutions were prepared for this purpose.

\subsection{Surface analysis of the host-tailored polymers}

The surface of the MI and NI modified silica beads was analyzed by SEM (Scanning electron microscope with X-ray microanalysis, JEOL JSM 35C/Noran Voyager). SEM observation was conducted with metal coating at $33 \mathrm{~Pa}$ and room temperature by using low vacuum mode. EDC analysis was carried out in several points of each material.

FTIR analysis was conducted in a Nicolet 6700 FTIR spectrometer. Infrared spectra were collected under room temperature/humidity control after background correction. The number of scans was 32 for both sample and background. $X$-Axis was wavenumber, ranging 525 to $4000 \mathrm{~cm}^{-1}$, and $Y$-axis was \% transmittance. Resolution was 4000 .

\subsection{Synthesis of host-tailored polymers}

About $100 \mathrm{mg}$ of aminopropyl silica was suspended in $1 \mathrm{~mL}$ of $10 \mathrm{mM}$ pipes buffer ( $\mathrm{pH} 5$ ) and the mixture was then degassed for $10 \mathrm{~min}$ at room temperature. The supernatant was removed after and $1 \mathrm{~mL}$ of $10 \mathrm{mmol} / \mathrm{L}$ PIPES buffer ( $\mathrm{pH}$ ) containing $1 \%$ of glutaraldehyde was added to the wet aminopropyl silica. Theresulting mixture was incubated for $12 \mathrm{~h}$ at room temperature. The silica particles were then repeatedly washed with distilled water and incubated nextin $1 \mathrm{~mL}$ of $2.5 \mathrm{mg} / \mathrm{mLMb}$ solution $(\mathrm{pH} 7,10 \mathrm{mmol} / \mathrm{L}$ MOPS, $1 \mathrm{~mol} / \mathrm{L} \mathrm{NaCl}$ ) for $3 \mathrm{~h}$ at $4{ }^{\circ} \mathrm{C}$. The resulting supernatant was replaced by $10 \mathrm{mmol} / \mathrm{L}$ MOPS buffer (pH7). After that, $2.5 \mathrm{~mL}$ of 3-APTMS and $25 \mathrm{~mL}$ of PTMS were added and the subsequent polymerization was carried out at room temperature, for $36 \mathrm{~h}$. The $\mathrm{Mb}$-imprinted silica was rinsed with water until the supernatant 
was clear. Then, $1 \mathrm{~mL}$ of $1 \mathrm{~mol} / \mathrm{L}$ oxalic acid was added to remove template. This step was carried out at room temperature and the incubation lasted $12 \mathrm{~h}$. Finally, the product was repeatedly washed with distilled water and dried in a dessicator.

The synthesis of the non-imprinted material (NI) was made by the same process as that of the imprinted material but without the template molecule.

\subsection{Preparation of the potentiometric sensors}

$\mathrm{Mb}$ selective membranes were prepared by mixing $210 \mathrm{mg}$ of PVC, $350 \mathrm{mg}$ of plasticizer $o$-nitrophenyloctyl (oNPOE) and $15 \mathrm{mg}$ of MI and NI modified silica beads. The mixture was stirred until the PVC was well moistened, and dispersed in $2.5 \mathrm{~mL}$ THF. These membranes were applied over the solid conductive supports and let dry for $24 \mathrm{~h}$. After that they were conditioned in $1 \times 10^{-7} \mathrm{~mol} / \mathrm{L}$ $\mathrm{Mb}$ solution. The electrodes were kept in this solution when not in use.

\subsection{Potentiometric procedures}

All potentiometric measurements were carried out at room temperature and under constant stirring. Increasing concentrations of $\mathrm{Mb}$ were obtained by transferring 0.0200 to $2.5 \mathrm{~mL}$ aliquots of $1.0 \times 10^{-4} \mathrm{~mol} / \mathrm{L} \mathrm{Mb}$ aqueous solution to a $100 \mathrm{~mL}$ beaker containing $20.0 \mathrm{~mL}$ of $1.0 \times 10^{-3} \mathrm{~mol} / \mathrm{L}$ of suitable buffer, of fixed $\mathrm{pH}$ and ionic strength. The potential readings of the stirred $\mathrm{Mb}$ solutions were measured at room temperature and recorded after stabilization to $\pm 0.2 \mathrm{mV}$. Between assays, the sensors were conditioned in $1 \times 10^{-5} \mathrm{~mol} / \mathrm{L} \mathrm{Mb}$ solution.

\subsection{Selectivity}

Potentiometric selectivity coefficients were assessed by the Matched Potential Method (MPM). The initial concentration of primary ion was set to $1.0 \times 10^{-6} \mathrm{~mol} / \mathrm{L}(a A)$ and the activity of the primary ion solution added into this was $4.0 \times 10^{-6} \mathrm{~mol} / \mathrm{L}\left(a A^{*}\right)$. The corresponding potential change was about $10 \mathrm{mV}$. The interference of Creat, Sac, Fru, Gat, Glt, Tia, Ala, and Gluc was assessed by adding small aliquots of the corresponding solutions into the primary ion solution of $a A$ until the same potential change was observed, i.e., until the increment of $10 \mathrm{mV}$ was reached, ensuring that the final concentration of primary ion was not altered by more than $5 \%$.

\subsection{Determination of binding capacities of MI materials}

Binding experiments were carried out by placing $5.0 \mathrm{mg}$ of MI washed particles in contact with $3.5 \mathrm{~mL} \mathrm{Mb}$ aqueous solutions ranging $0.02-2.0 \mathrm{mM}$. The mixtures were oscillated for $20 \mathrm{~h}$ at room temperature and the solid phase separated by centrifugation (3000 rpm, $10 \mathrm{~min}$ ). Mb concentrations in the supernatant were measured by UV/vis spectrophotometry (at $410 \mathrm{~nm}$ ) against calibration graph with $\mathrm{Mb}$ standard solutions.

\section{Results and discussion}

\subsection{Mb artificial antibody assembly}

In MI the template recognition capability is defined by the kind(s) of sites or functional groups present in the polymerizable materials employed for their generation. Considering specifically the protein imprint, their hydrophobic interactions with silanes surfaces may play an important role, as supported by previous observations (Cha et al., 1999; Venton and Gudipati, 1995). On the other hand, surface imprint is much more effective than bulk procedures when sensory surfaces are to be prepared.
Thus, aminopropyl silica was employed as the base material to assemble the $\mathrm{Mb}$ artificial antibody on its surface. Nanopored silica was selected for this purpose because it has a large surface area and known capacity to adsorb Mb (Artyomova et al., 1986; Wu and Walters, 1988). The surface of the silica beads was modified following the scheme in Fig. 1. It consisted of three different stages: (i) template immobilization on the bead surface, (ii) filling vacant places with suitable functionalities and hard material, and (iii) template removal, leaving behind its imprinted site with rebinding properties.

$\mathrm{Mb}$ was immobilized by reaction between its amine groups and the aldehyde function on the surface of the beads, with subsequent formation of an imine bond. Aldehyde groups were introduced on the external surface of the beads by reacting aminopropyl silica with glutaraldehyde.

The organic silane monomers3-aminopropyltrimethoxysilane (APTMS) and propyltrimethoxysilane (PTMS) were selected to fill with solid-state materials the free space around the template. They polymerize under mild conditions which contributed to keep the templateconfiguration unaltered.

$\mathrm{Mb}$ imprinted material resulted from the template removal (Wanga et al., 2008). Strong acids had to be used to break the silica-protein covalent bonds. Thus, the material was incubated in $1 \mathrm{~mol} / \mathrm{L}$ oxalic acid for $12 \mathrm{~h}$ (Shiomi et al., 2005). Several washing steps were made after with $10 \mathrm{mmol} / \mathrm{L}$ MOPS buffer to remove the protein released from the beads and increase the inherent $\mathrm{pH}$ from 1.2 to 8.0. The modified beads were conditioned in this buffer before carrying out binding experiments.

\subsection{Surface analysis of the host-tailored polymers}

\subsubsection{SEM/EDC analysis}

The silica beads had an average diameter of 15-40 $\mathrm{nm}$ and were mainly composed by Si and O, before surface modification. Clearly, the imprinting effect could not be proven by means of SEM, because MI and NI materials were similar. However, the chemical modification of the surface was easily detected. The diameter of the beads increased up to $125.6 \mathrm{~nm}$, measured when the original material was magnified by $400,000 \times$. This was consistent with the EDS spectra (Fig. 2). Both MI and NI materials showed similar composition, with $\mathrm{N}$ and $\mathrm{C}$ (besides $\mathrm{Si}$ and $\mathrm{O}$ ). These atoms came from the APTMS and PTMS monomers used for the sol-gel based imprinting.

\subsubsection{FTIR analysis}

Structural changes of the silica beads and its composites were confirmed by FTIR spectra (Fig. 3). The IR spectrum for non-modified silica beads showed significant bands at 920 and $1075 \mathrm{~cm}^{-1}$, revealing the medium intensity absorption bands from theSi-OHandSi-O-Si vibrations, respectively.

Since the chemical modification of the silica beads also employed sol-gel based materials, the above peaks were also in the spectra of FTIR surface analysis of the MI and NI modified beads. These spectra also pointed out a small increase of the $\mathrm{G} O \mathrm{O}$ band, approximately at $1620 \mathrm{~cm}^{-1}$. Since the carbonyl bears a highly absorbing bond, it is likely that only a very small part of the surface had unreacted $\mathrm{C}=$ o groups. The broad absorption low intensity band lying between 3000 and $3500 \mathrm{~cm}^{-1}$ was probably a combined result of the $\mathrm{N}-\mathrm{H}$ stretch of the amine groups (introduced through polymerization of APTMS) and the O-H stretch of a small number of alcohol groups generated by alkoxide hydrolysis.

\subsection{Rebinding properties of the imprinted materials}

In practical terms, the MI materials should have a high affinity for the template to which they were designed. This may be assessed by rebinding experiments. For this purpose, fixed amounts of MI 


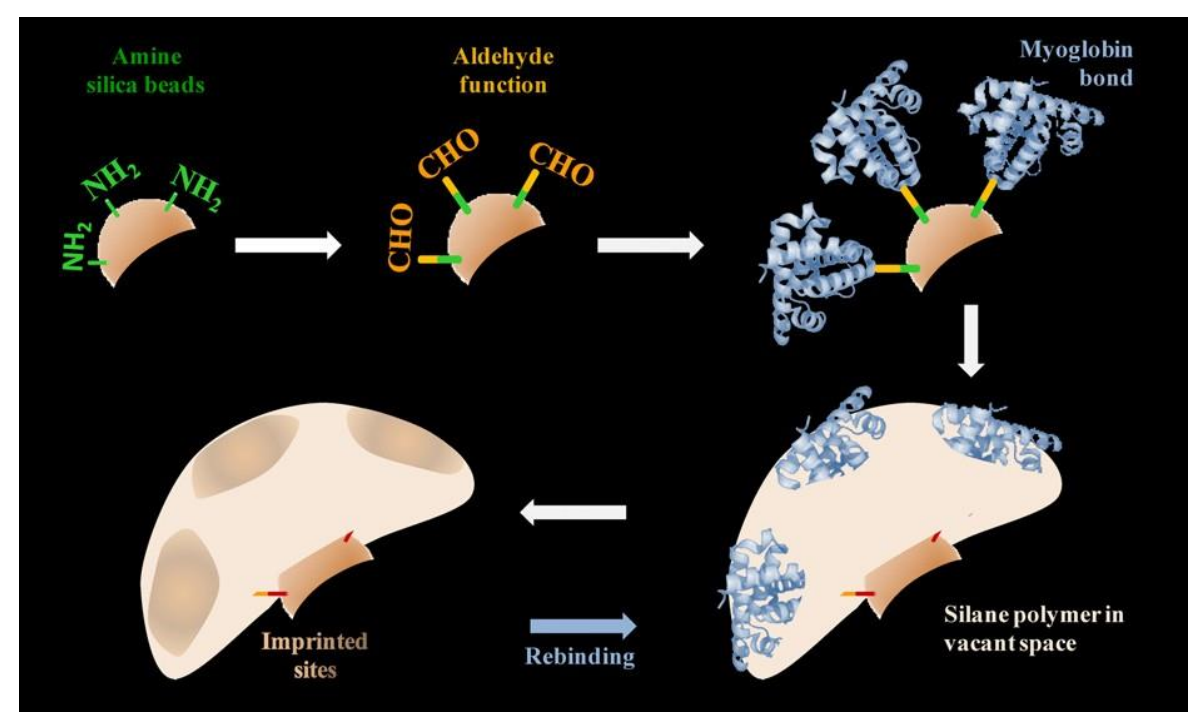

Fig. 1. Assembly of the MI materials.

materials were incubated with different concentrations of Mb until the equilibrium was reached (Connors, 1987; Shea et al., 1993). The resulting binding capacity was calculated by Eq. (1):

$$
Q=\frac{\mu \mathrm{mol}(\mathrm{OXY} \text { bound })}{\mathrm{g}(\mathrm{MIP})}=\frac{\left(C_{i}-C_{f}\right) V_{s} \times 1000}{M_{\mathrm{MIP}}}
$$

where $Q$ was binding capacity of $\mathrm{MI}(\mu \mathrm{mol} / \mathrm{g}), C_{i}$ the initial Mb concentration $(\mu \mathrm{mol} / \mathrm{mL}), C_{f}$ the final $\mathrm{Mb}$ concentration $(\mu \mathrm{mol} / \mathrm{mL})$, $V_{s}$ the volume of solution tested $(\mathrm{mL})$, and $M_{\mathrm{MI}}$ the mass of dried polymer(mg).

The binding capacities were plotted against $\mathrm{Mb}$ initial concentrationintheincubationmedium(Fig.4A). Theresulting adsorption data showed that the binding capacity of imprinted polymer increased with the increasing of the initial concentration of $\mathrm{Mb}$, but it reaches to saturation when high concentrations of template are present. The binding data was used to carry out Scatchard anal- ysis, providing important information on binding properties of the imprinted particles (Sellergren, 2001). The Scatchard equation,

$$
\frac{Q}{C_{\text {free }}}=\frac{Q_{\max }-Q}{K_{d}}
$$

was applied for this purpose, where $Q$ is the binding capacity; $C_{\text {free }}$ the free analytical concentration at equilibrium $(\mu \mathrm{mol} / \mathrm{L}) ; Q_{\max }$ is the maximum apparent binding capacity; and $K_{d}$ is the dissociation constant at binding site. The equilibrium dissociation constant was calculated from the slopes and the apparent maximum number of binding sites from the $y$-intercepts in the linear plot of $Q / C_{\text {free }}$ versus $Q$.

Scatchard plot of the MI consisted of two distinct straight lines (Fig. 4), inferring the existence of high and low affinity populations of binding sites (Sellergren, 2001). $K_{d 1}$ and $Q_{\max }$ were, respectively, $0.21 \mu \mathrm{mol} / \mathrm{L}$ and $49 \mu \mathrm{mol} / \mathrm{g}$ for the high affinity binding sites, and $12 \mu \mathrm{mol} / \mathrm{L}$ and $163 \mu \mathrm{mol} / \mathrm{g}$ for the low affinity binding sites. The lower affinity binding resulted from non-specific interactions while the higher affinity came from the interaction between specific sites

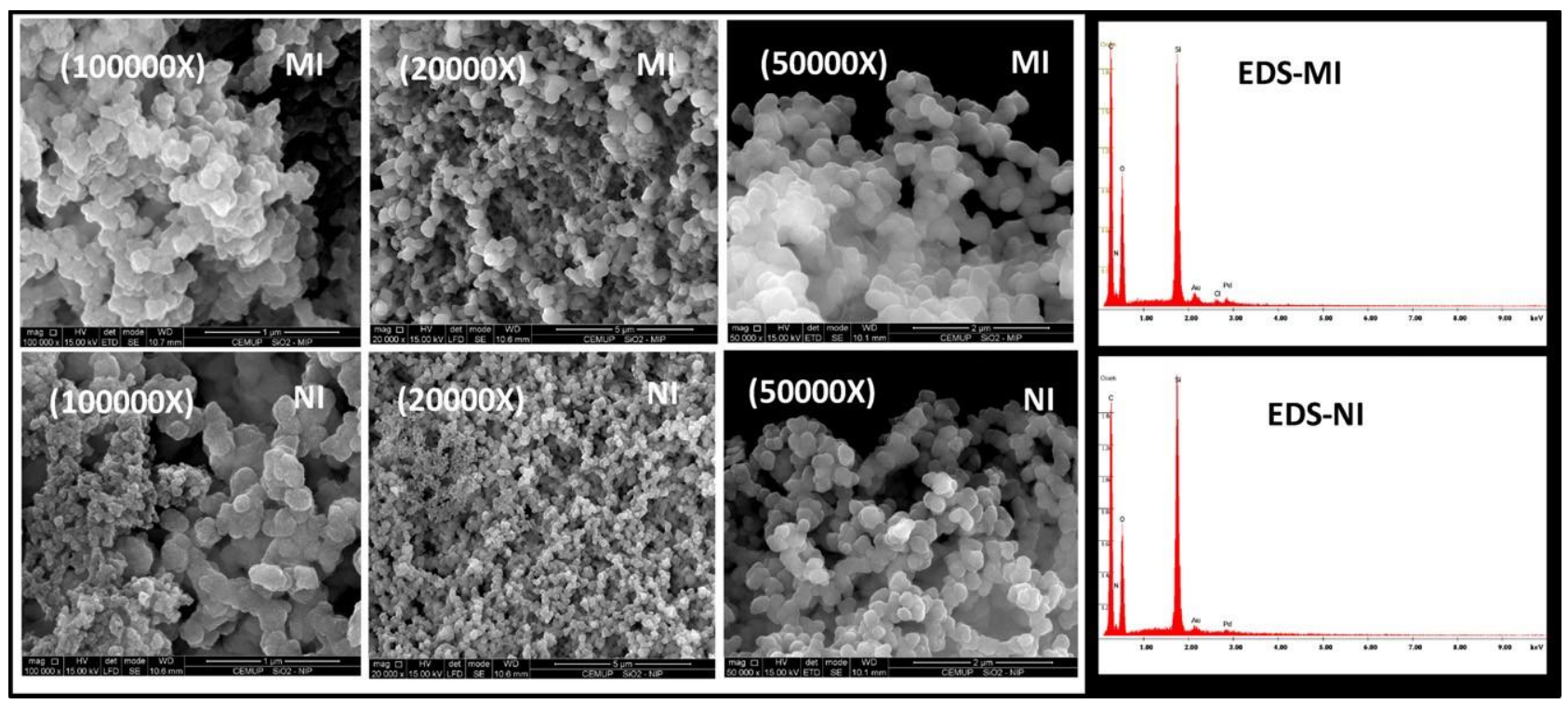

Fig. 2. SEM and EDC analysis of MI (A) and NI (B) modified silica beads immobilized in tape carbon. 


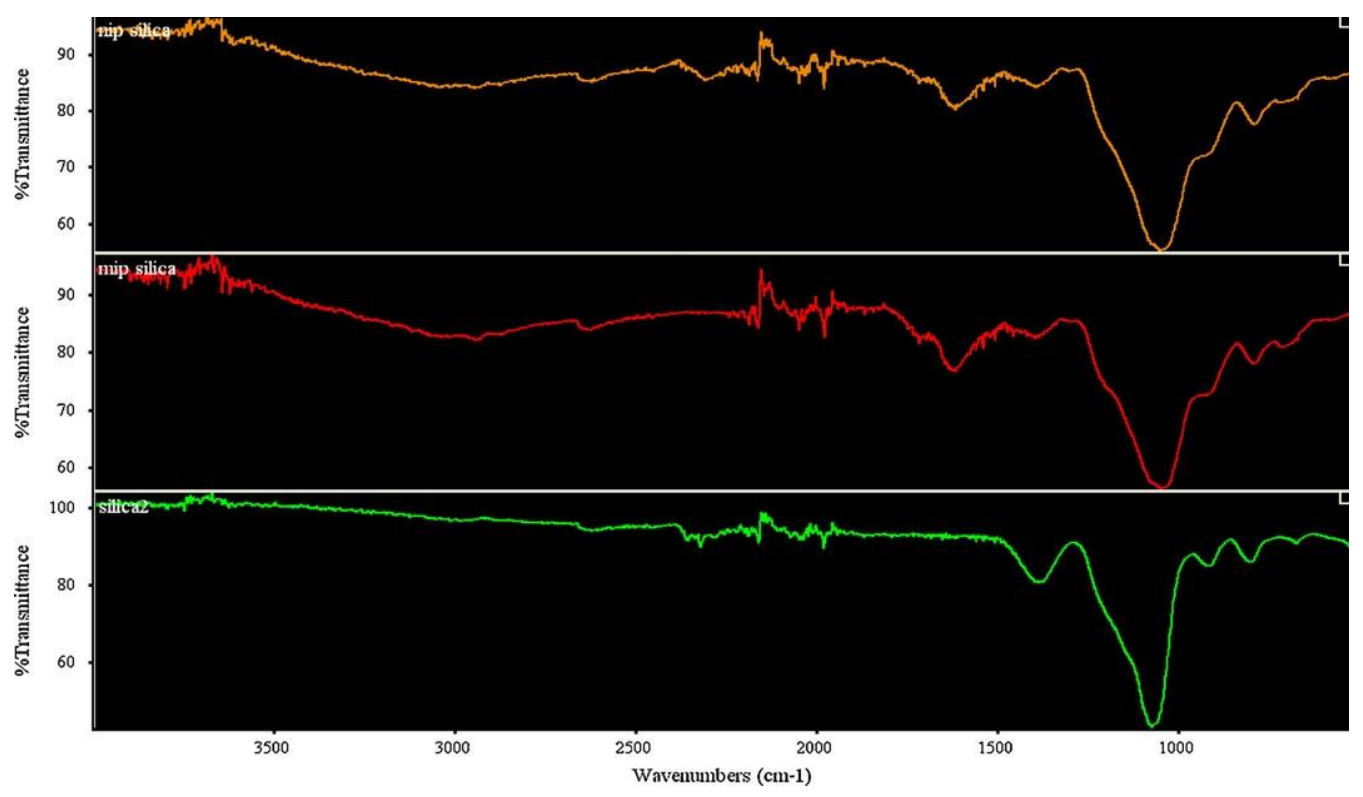

Fig. 3. FTIR spectra of modified (MI or NI) and unmodified silica beads.

of the artificial antibody and the template, leading to a stereochemical recognition of the analyte.

\subsection{MI and NI acting as ionophores}

$\mathrm{Mb}$ sensors were prepared by dispersing the active ingredients (MIor NI particles) in a high dielectric constant plasticizer and PVC. Evaluation of theirmainanalytical featuresfollowed theIUPACrecommendations (Umezawa et al., 2000), and was carried out in batch conditions.

For NI sensors, the emfs changed linearly with log $[\mathrm{Mb}]$ for concentrations down to $1 \times 10^{-6} \mathrm{~mol} / \mathrm{L}$, displaying detection limits within the $10^{-7} \mathrm{~mol} / \mathrm{L}$ decade. On the contrary, the potentiometric sensor prepared with NI particles was unable to produce a linear response, no matter the $\mathrm{pH}$ of the test solution (Fig. 5). These results indicated that the non-specific places of interaction (the only kind that exists on NI particles) between the template and the polymer were not the main responsible for the potentiometric response. Only monomers polymerized in the presence of a template could have imprinted cavities, enabling the specific interaction between the template and the sensor. Therefore, the potentiometric response observed for the MI electroactive materials was mainly due to a topographic recognition of the analyte at the imprinted cavities.

\subsection{Effect of $p H$}

Electrodes with MI particles were calibrated in different $\mathrm{pH}$ conditions $(3,4,5,6$ and 9). The results obtained are presented in Fig. 5. Linear behavior was observed down to $3.5 \times 10^{-6}, 1.2 \times 10^{-6}$, $1.2 \times 10^{-6}, 1.2 \times 10^{-6}$, and $3.8 \times 10^{-6} \mathrm{~mol} / \mathrm{L}$ of $\mathrm{Mb}$, with anionic slopes of $-178.7,-65.9,-25.5,-12.5$, and $+8.2 \mathrm{mV} /$ decade and detection limits of $81.3,13.5,10.7,2.2$, and $5.4 \mu \mathrm{g} / \mathrm{mL}$, respectively. The squares correlation coefficients were always $>0.990$. The electrodes were also tested in $\mathrm{pH} 7.5$ but there was no linear response observed.

Essentially, the slopes were anionic below $\mathrm{pH}<7.5$ and turned out cationic for $\mathrm{pH} 9.0$. These results suggested that $\mathrm{Mb}$ was negatively charged below $7.5 \mathrm{pH}$ and positively charged above that value. However, this is the opposite behavior expected from the isoelectric point for $\mathrm{Mb}$, equal to 7.2. Myoglobin has many ionizable functional groups on the outer surface that may interact with

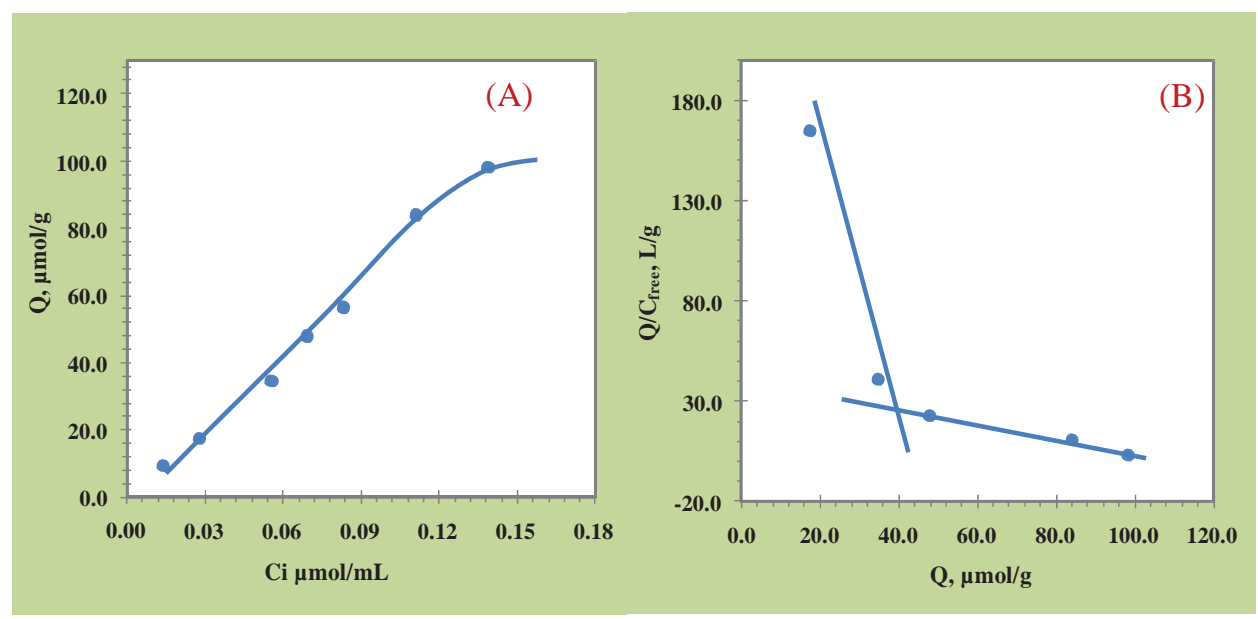

Fig. 4. Binding isotherm (left) and Scatchard plot (right) for Mb imprinted materials. 


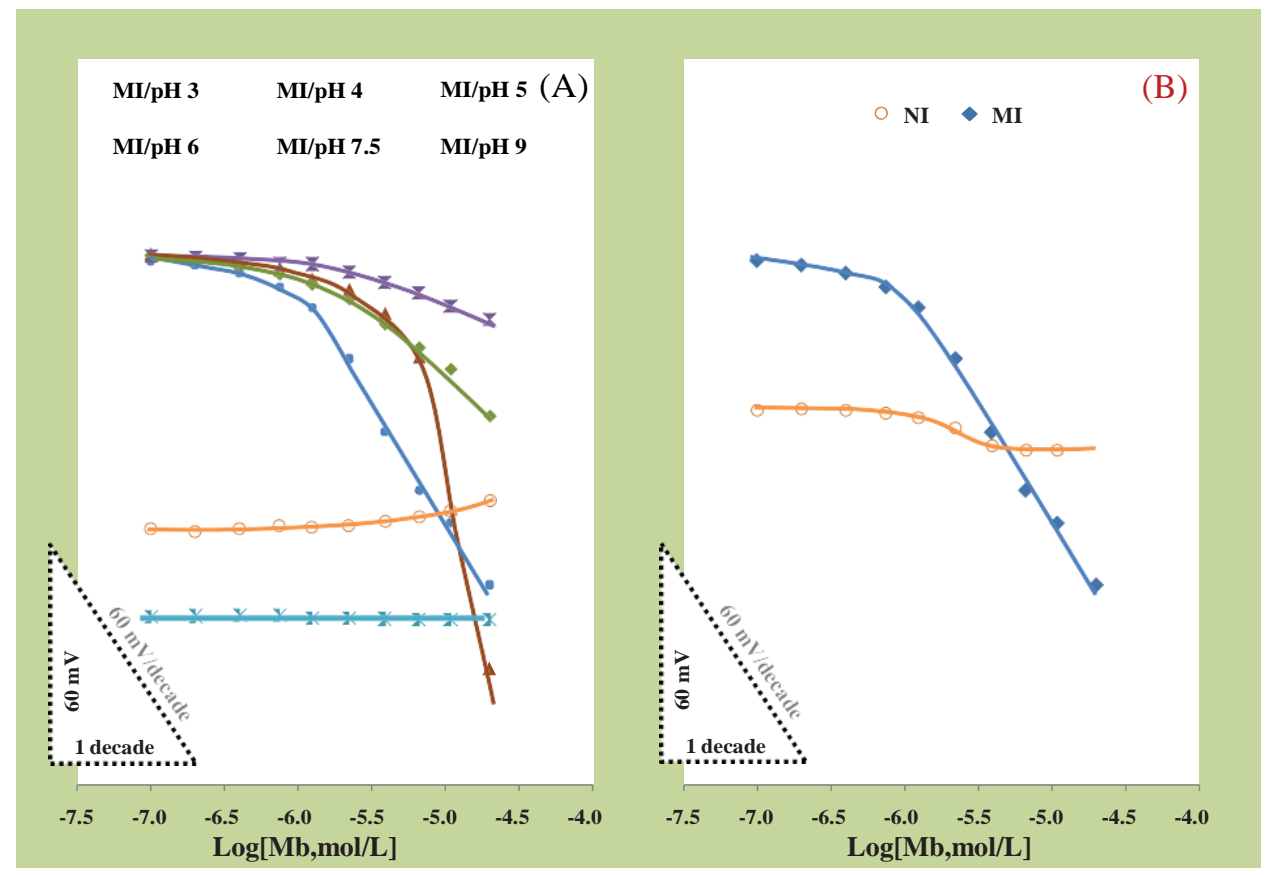

Fig. 5. Calibration curves of $\mathrm{MI}$ based sensors in different $\mathrm{pHs}$ (A) and MI and NI sensors in $\mathrm{pH} 4$ (B).

the membrane. The closest idea to explain the negative behavior of these selective electrodes under acidic media is that the protein is approaching the membrane by its negatively charged groups, which is favored by the positively charged $\mathrm{H}^{+}$hanging close to the membrane and creating a positive environment. Apparently, it seems that the exceeding $\mathrm{H}^{+}$in acidic medium is binding to the electrode surface and contributing to the detection of a negatively charged portion of $\mathrm{Mb}$.

In terms of analytical application, a $\mathrm{pH}$ of 4 was selected for further studies. This was the only condition where the sensor displayed a near-Nernstian behavior, assuming that Mb was singly charged under this condition.

\subsection{Response time and lifetime}

The time required to achieve a steady potential response $( \pm 3 \mathrm{mV})$ in $1.0 \times 10^{-3} \mathrm{~mol} / \mathrm{L} \mathrm{Mb}$ solutions with a rapid 10 -fold increase in concentration was $<15 \mathrm{~s}$. Low potential drift and long-term stability were observed within calibrations made in consecutive days. In general, detection limits, response times, linear range and calibration slopes were reproducible within $\pm 5 \%$ of their original values over a period of at least 5 weeks.

\subsection{Sensor selectivity}

The selectivity of an ISE depends on the selectivity of the template/ionophore binding and on the standard free energies of the

respective ions in the aqueous and organic phases (Bakker and Pretsch, 2001). Thus, a high selectivity suggests the use of ligands that strongly bind the preferred ion and only weakly all the others.

The selectivity behavior of Mb ISEs was observed by calculating logarithmic potentiometric selectivity coefficients, $\log K^{\mathrm{POT}}$. These coefficients defined the ability of an ISE to differentiate a particular (primary) ion from others (interfering species). The matched potential method (MPM) was used for this study (Umezawa et al., 2000 ), where $K^{\mathrm{POT}}$ is defined as the activity (concentration) ratio of primary and interfering ions that give the same potential change under identical conditions (Eq. (3)). A known activity $\left(a A^{k}\right)$ of the primary ion solution is added first into a reference solution that contains a fixed activity $(a A)$ of primary ions, and the corresponding potential change $(E)$ is recorded. Next, a solution of an interfering ion is added $(a B)$ to the reference solution until the same potential change (E) is observed. The change in potential produced at the constant background of the primary ion must be the same in both cases.

$$
K_{A, B}^{\mathrm{POT}}=\frac{a A^{\prime}-a A}{a B}
$$

Creat, Sac, Fru, Gal, Glu, Glt, Ala, and Tia were tested as possible interfering species in biological samples. Overall, $\log K^{\mathrm{POT}}$ ranged from -0.69 to 2.50 and the lowest value $(-2.5)$ was obtained for creatinine, a biological metabolite. Different amino acids interfered in a similar way, leading to $\log K^{\mathrm{POT}} \approx 1.3$. The relative selectivity degree of sugar interference was Fru $<\mathrm{Gal}<\mathrm{Glu}<\mathrm{Sac}$, with $\log K^{\mathrm{POT}}$ varying from -2.45 to -0.69 . The interference from Tia $\mathrm{p}_{\mathrm{p}}$ tamin $\mathrm{B}_{1}$, a sulfur-containing vitamin, was negligible, with $\log K=-0.8$.

In general, the recorded values reported negligible interference for all species tested (Table 1). The interference of hemoglobin was also tested for concentrations ranging from 700 to $70,000 \mathrm{ng} / \mathrm{mL}$ and found negligible.

\subsection{Mb assay}

A real application requires previous pre-concentration procedures, that can reach up to $1000 \times$ the concentration in the sample. This has been already tested and described in theliterature(Nesbitt,

Table 1

Potentiometric selectivity coefficients of NI Mb sensors.

\begin{tabular}{lc}
\hline Interfering species & $\log K^{\mathrm{POT}}$ \\
\hline Creatinine & -2.50 \\
Sucrose & -0.69 \\
Fructose & -2.45 \\
Galactose & -1.24 \\
Sodium glutamate & -1.37 \\
Alanine & -1.27 \\
Glucose & -1.11 \\
Tiamine & -0.81 \\
\hline
\end{tabular}


2005). Thus, the present method has presumably applied to those concentrated samples.

The method was applied to determine $\mathrm{Mb}$ in spiked samples ranging from 107.9 to $321.8 \mu \mathrm{g} / \mathrm{mL} \mathrm{Mb}$. A good agreement was found between added and found amounts of $\mathrm{Mb}$. Results of the potentiometric analysis conducted in steady state showed recoveries ranging from 93.8 to $107.5 \%$ with an average relative standard deviation of $1.8 \%$. The relative error was ranging from 5.70 to 6.96 with an average relative standard deviation of $0.02 \%$.

\subsection{Impedimetric analysis}

Detection capability may be improved by changing the technique and the electrode design. Since the potentiometric sensitivity is regulated by the Nernst equation, no further improvements may be obtained by this technique, and impedance was selected to improve the present proposal. Impedance spectroscopy tests the electric features of surface modifies electrodes. Gold-screen printed electrodes modified by the same imprinting procedure were used in order toimprovesignal/noiseratio.

Impedimetric analysis was conducted on modified gold-screen printed electrodes. The electrode modification was made by applying the same PVC membrane over the gold surface. Impedance measurements were performed in SSPE buffer ( $\mathrm{pH}$ 7.4) including $5.0 \mathrm{mmol} / \mathrm{L}[\mathrm{Fe}(\mathrm{CN}) 6]^{3-}$ and $5.0 \mathrm{mM}[\mathrm{Fe}(\mathrm{CN}) 6]^{4-}$ at a standard potential of $0.225 \mathrm{~V}$, with a number of frequencies equal to 50 and of amplitude $0.01 \mathrm{~V}$. The frequency range was $0.1-100 \mathrm{kHz}$. The charge-transfer resistance calculated from the semicircle of the Nyquist plot decreased with concentration (Fig. S1, left). This behavior was probably correlated to the charge transfer of $\mathrm{Mb}$ across the membrane, which was consistent with the large dipole moment of the protein. Overall, this behavior could be used for concentration-dependent impedance measurements. The impedance data were fitted to an equivalent circuit using the implemented ANOVA software (Fig. S1, right). The sensor displayed better features in terms of linear range compared to the graphite electrodes, decreasing the lower limit for linear response from $1.24 \times 10^{-6} \mathrm{~mol} / \mathrm{L}$ to $3.71 \times 10^{-7} \mathrm{~mol} / \mathrm{L}$.

\section{Conclusions}

MI technology was successfully employed to produce biomimetic $\mathrm{Mb}$ sensors for potentiometric transduction with similar to host-guest interactions. The shape of $\mathrm{Mb}$ was tailored by attaching it covalently to a silane surface and filling the surroundings by polymerization of 3-APTMS and PTMS monomers. The covalent bond to $\mathrm{Mb}$ was broken and the corresponding site showed rebinding capabilities, indicating that the template was stereochemically recognized.

Only imprinted organic materials were capable to discriminate $\mathrm{Mb}$ from other organic compounds in aqueous media in potentiometric assays. The corresponding sensors offered simplicity in designing; low response time, good precision, high accuracy, low limit of detection and good selectivity. The proposed method is simple, of low cost, precise, accurate and inexpensive, and may turn out, in a near future, an alternative method for screening $\mathrm{Mb}$ in point of care.

\section{Acknowledgement}

One of the authors (FTCM) gratefully acknowledges Fundação para a Ciência e Tecnologia for the financial support (PhD grant reference SFRH/BD/66735/2009).

\section{Appendix A. Supplementary data}

Supplementary data associated with this article can be found, in the online version, at doi:10.1016/j.bios.2011.05.045.

\section{References}

Adams, J.E., Miracle, V.A., 1998. Am. J. Crit. Care 6, 418-423.

Alan, W.H., Skillena, A.W., Argent, N.B., 1992. Clin. Chim. Acta 31, 197-207. Artyomova, A.A., Voroshillova, O.I., Yu, S.N., Khokhlova, T.D., 1986. Adv. Colloid Interface Sci. 25, 235-248.

Bakker, E., Pretsch, E., 2001. Trends Anal. Chem. 21, 11-19.

Billah, M., Hays, H.C.W., Millner, P.A., 2008. Microchim. Acta 160, 447-454

Boesken, W.H., Boesken, S., Mamier, A., 1977. Exp. Med. 171, 71-78.

Cha, J.N., Shimizu, K., Zhou, Y., Christiansen, S.C., Chmelka, B.F., Stucky, G.D., Morse D.E., 1999. Proc. Natl. Acad. Sci. USA 96, 361-365.

Connors, K.A., 1987. Binding Constants. The Measurement of Molecular Complex Stability. John Wiley \& Sons, New York.

Fresnea, A., 1964. Life Sci. 3, 1201-1206.

Gunda, N.S.K., Mitra, S.K., 2010. Biomicrofluidics 4, 014105.

Han, D., Mcmillin, K.W., Godber, J.S., 1994. J. Food Sci. 59, 1279-1282.

Kelner, M.J., Alexander, N.M., 1985. Clin. Chem. 31, 112-114.

Li, J., Liu, D., Tian, L., Yang, L., Hu, N., 2009. Electroanalysis 21, 1555-1658.

Malone, M.R., Masson, J.F., 2005. Proc. SPIE, 6007 , doi:10.1117/12.630744.

Masson, J.F., Battaglia, T.M., Khairallah, P., Beaudoin, S., Books, K.S., 2007. Anal. Chem $79,612-619$.

Mayer, B.M., Kohlbacher, O., Reinert, K., Sturm, M., Gröpl, C., Lange, E., Klein, C., Huber, C.G., 2006. J. Proteome Res. 5, 414-421.

Maltseva, V.G., Ziminaa, T.M., Khvatova, A.B., Belenkii, B.G., 1987. J. Chromatogr. 416 45-52.

Montague, C., Kircher, T., 1995. Am. J. Clin. Pathol. 4, 472-476.

Modi, S., Shedbalkar, V.P., Behere, D.V., 1989. Indian J. Biochem. Biophys. 26, 84-86. Moreira, F.T.C., Kamel, A.H., Guerreiro, J.R.L., Sales, M.G.F., 2010. Biosens. Bioelectron. $26,566-574$.

Nesbitt, A.C., Lo, J.T.M., Yeung, K.K.C., 2005. J. Chromathogr. A 1073, 175-180.

Plebani, M., Zaninotto, M., 1999. Int. J. Clin. Lab. Res. 29, 56-63.

Powell, S.C., Friedlander, E.R., Shihabi, Z.K., 1984. J. Chromatogr. 28, 87-92.

Sarkara, M., Mandala, C., 1985. J. Immunol. Methods 83, 55-60.

Sellergren, B., 2001. Molecularly Imprinted Polymers. Elsevier, The Netherlands.

Schuder, S., Wittenberg, J.B., Haseltine, B., Wittenberg, B.A., 1979. Anal. Biochem. 92, $473-481$.

Shea, K.J., Spivak, D.A., Sellergren, B., 1993. J. Am. Chem. Soc. 115, 3368-3369.

Shiomi, T., Matsui, M., Mizukami, F., Sakaguchi, K., 2005. Biomaterials 27 (27) 5564-5571.

Singh-Zocchi, M., Hanne, J., Zocchi, G., 2002. Biophys. J. 83, 2211-2218.

Umezawa, Y., Bühlmann, P., Umezawa, K., Tohda, K., Amemiyap, S., 2000. Pure Appl. Chem. 72, 1851-2082.

Venton, D.L., Gudipati, E., 1995. Biochim. Biophys. Acta 1250, 117-125.

Wanga, Y., Zhoub, Y., Sokolova, J., Rigasc, B., Kalle, L., Rafailovicha, M., 2008. Biosens. Bioelectron. 24, 162-166.

Wu, D., Walters, R.R., 1988. J. Chromatogr. 458, 169-174.

WHO, World Health Organization, Health Topics, Cardiovascular Diseases, www.who.int/topics/cardiovascular_diseases/en/ (accessed on April, 2011). Zhang, H.M., Li, N.Q., 2001. Bioelectrochemistry 53, 97-101. 